\title{
Evaluating a model of global psychophysical judgments for brightness: I. Behavioral properties of summations and productions
}

\author{
RAGNAR STEINGRIMSSON \\ University of California, Irvine, California
}

\begin{abstract}
Steingrimsson and Luce (2005a, 2005b, 2006, 2007) evaluated Luce's (2002, 2004) proposed psychophysical theory of loudness and found it substantially supported. The aim of the present research is to begin an extension of this research to brightness. Luce's $(2002,2004)$ theory deals with the global percept of subjective intensity, in which there is a psychophysical function $\Psi$ that maps pairs of physical intensities onto positive real numbers and represents, in an explicit mathematical way, subjective summation and a form of ratio production. These representations derive from a number of behavioral properties, including certain plausible background assumptions. In three experiments involving the subjective perception of luminance, brightness, the key behavioral properties of summation over the two eyes, and a form of generalized ratio production are empirically evaluated. Considerable support is reported for particular forms of $\Psi$ separately for summations and ratio productions.
\end{abstract}

In a series of four articles, Steingrimsson and Luce (2005a, 2005b, 2006, 2007) evaluated Luce's (2002, 2004) global psychophysics theory of loudness. The results provided broad support for the branch of the theory in which ears are not assumed to be behaviorally completely alike (the biased or asymmetric case). The main aim of this article is to begin an analogous series of evaluations of the theory interpreted for the brightness domain. The article is organized as follows:

In the first section, I summarize the relevant portions of Luce's $(2002,2004)$ theory of global psychophysical judgments. In the tradition of the axiomatic approach, the theory presents non-domain-specific elements (primitives), which are interpreted in the context of brightness. To place this article in that context, the ongoing experimental program is outlined. Three experiments are presented, in which three behavioral properties, or axioms, are empirically evaluated. What about the theory can be concluded on the basis of the results is discussed. The article's subject matter and overall conclusions are summarized, and the consequences of those conclusions for subsequent work are outlined. In the appendices, I address the historical background of the work, the axiomatic psychophysical approach, and how Luce's (2002, 2004) model fits in with previous and related work. To accommodate a variety of readers, these are presented as separate appendices, to be read as needed.

Psychological experiments on brightness are carried out using a computer monitor and a keyboard, with which participants, seated in dark room, can alter the luminance of stimuli in accordance with instructions. The stimuli consist of squares of achromatic light - see Figure 1A for an example. The tasks consist of making a certain stimulus equal in brightness to a standard (matching) or to some proportion of the standard - for example, two times as bright as the standard (called either ratio or magnitude production). In some cases, a stereoscope - see Figure $1 \mathrm{~B}$ - is used to generate the stimuli.

Whatever physical signal attribute is taken to correspond to the sensation that we call brightness, it seems crucial that it be defined independent of a hypothetical observer (otherwise, the physical stimulus is mutable by a change of observer, a feature not accorded to variables such as temperature, volume, length, etc.). Here, brightness and its change are taken to be the sensations elicited by a luminance and changes of it. This functional definition of brightness accords with a great many other experiments (see Grossberg \& Kelly, 1999, and Ding \& Sperling, 2006). Intuitively, brightness is the sensation that changes when, for example, one varies the luminance setting on a computer monitor or goes from outside on a sun-filled day into a movie theater.

It should be stressed that since Luce's $(2002,2004)$ theory is non-domain-specific, it is applicable to any domain in which its primitives may be defined. Thus, the theory is not one of binocular brightness, but rather it is applied to binocular brightness. For that reason, when I refer to the primitives in the context of binocular brightness, it is only because of the specific application of the theory here to that domain (for further details, see Appendix A). 
A

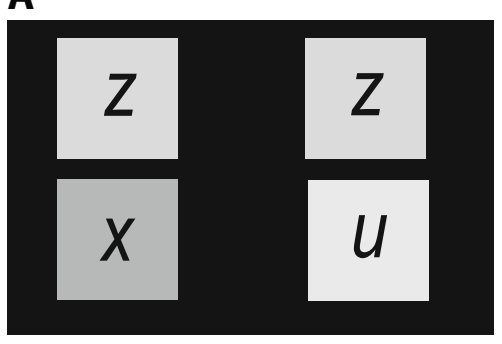

B

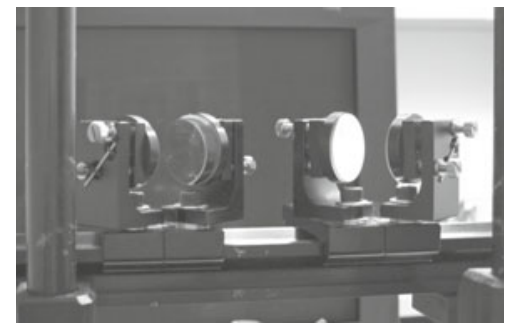

C

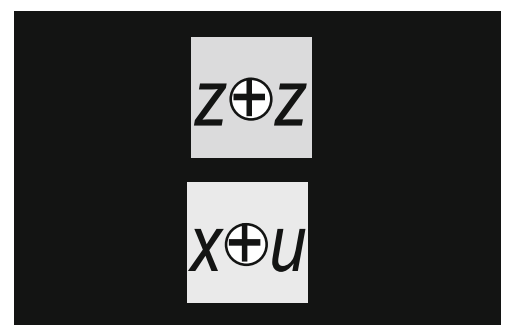

Figure 1. Stimuli displayed on a monitor (A), or viewed through a stereoscope (B), produce the subjective percept seen by the respondents (C). The $x, u$, and $z$ values are luminance.

\section{SUMMARY OF THEORY AND INTERPRETATION IN BRIGHTNESS}

\section{Primitives}

The first step in the axiomatic approach is the specification of the theory's primitives. Interpreting these in the context of brightness will determine the stimuli, as well as how they may be manipulated (for details, see the first section of Appendix A).

Joint presentations. The first primitive is the set of ordered pairs $(x, u)$, where $x$ and $u$ correspond to physical intensities. Our interpretation of this primitive in the visual domain is a pair of squares of achromatic light (RGB settings of equal values) having the intensities $x$ and $u$ presented simultaneously to the left and right eyes, respectively. Technically, this is achieved using a stereoscope (see Figure 1B), as is detailed in the Apparatus section.

Ordering. The second primitive, $\succsim$, is the ordering of stimuli: The formalism $(x, u) \succsim(y, v)$ means that the stimulus $(x, u)$ is judged to be at least as bright as $(y, v)$. The indifference relation $\sim$ is defined by $(x, u) \sim(y, v)$ if and only if both $(x, u) \succsim(y, v)$ and $(y, v) \succsim(x, u)$ hold. The symbols $\succsim$ and $\sim$ are used rather than $\geq$ and $=$ because the latter refer to ordering of real numbers, whereas the former refer to psychological judgments; this means that $\succsim$ behaves in a manner similar to the ordering $\geq$ of real numbers. Moreover, it is assumed that the ordering agrees with physical intensity in the sense that if the intensity of the stimulus received by one eye is held constant, the binocular brightness varies monotonically with an intensity increase or decrease in the other eye.

Monotonicity and Fechner's paradox. The monotonicity of ordering has been demonstrated empirically by, for example, Levelt (1965). Figure 2 depicts the equibrightness curve produced from data by a respondent who adjusted the luminance in one eye to match a standard while the experimenter varied the luminance in the other eye.

The monotonicity assumption holds for all but the conditions in which the luminance in one eye is very small relative to that in the other eye. This condition creates the well-known Fechner's paradox: When the luminance ratio of the left to right (or vice versa) eye falls below a certain level, the subjective sense of brightness increases (Fechner, 1861); less energy gives rise to a sensation of increased intensity. In Figure 2 this occurs when the luminance in one eye is $13 \%$ less than that in the other eye
(Levelt's [1965] stimuli were structurally similar to those used here).

Luce's $(2002,2004)$ theory does not extend to the stimulus condition in which Fechner's paradox occurs. However, this does not present an important limitation. The crucial observation is that in binocular vision, the left and right eyes share $70 \%-80 \%$ of the visual input-more when looking into the distance. For this reason, it is rare for a natural binocular stimulus to exhibit the properties required to give rise to Fechner's paradox. In fact, as far as I know, Fechner's paradox has been demonstrated only by artificially separating the luminance sources to the left and right eyes. Since the theory under evaluation is a description of behavior, its not extending to this extreme viewing condition does not present a substantial limitation.

Matching operation. A standard method in psychophysics (see Appendix B and, e.g., S. S. Stevens, 1975, for a comprehensive discussion) is to match a stimulus to a standard. This method is used here, and it is formulated as

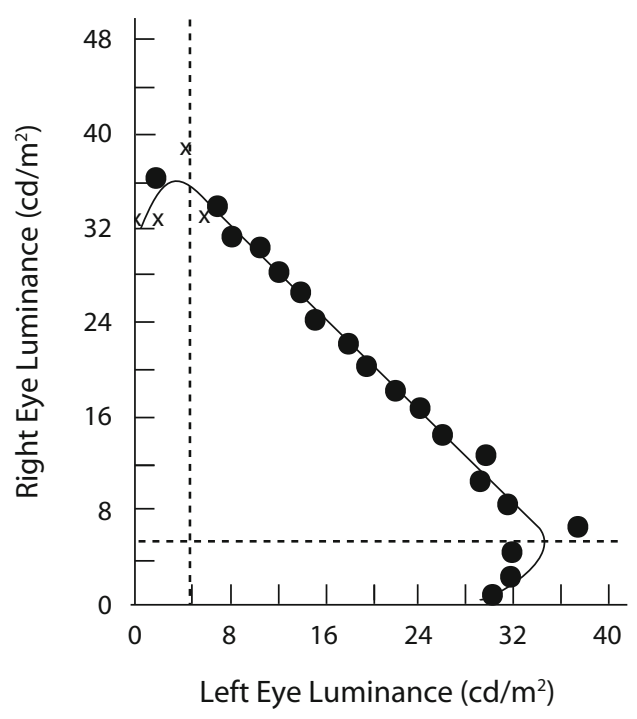

Figure 2. Equal-brightness curve for an observer. The experimenter adjusts the luminance in one eye, and the respondent adjusts that in the other to maintain a match to a standard. From "Binocular Brightness Averaging and Contour Information," by W. J. M. Levelt, 1965, British Journal of Psychology, 56, pp. 1-13, Figure 5. Copyright 1965 by the British Psychological Society. Adapted with permission. 
follows. Each joint presentation $(x, u)$ can be matched by $(z, z)$ - that is, formally,

$$
(x, u) \sim(z, z) .
$$

This is referred to theoretically as a symmetric match. Operationally, it will be referred to as brightness matching or just matching for short. It is convenient to express matching using an operator notation:

$$
x \oplus u:=z,
$$

where the notation $A:=B$ means that $A$ is defined by $B$, and $z$ is defined by Equation 1. One can prove that $\oplus$ is, technically, a mathematical operator, which is referred to as the summation operator.

Note that $(x, u)$ refers to a joint presentation of a signal pair. However, $x \oplus u$ refers to the cyclopic image that results. Since the cyclopic image results from the neural system's combination of the two input signals, I will refer to this as a subjective summation of the two signals (nothing is hypothesized about how this summation is accomplished). In practice, the notations are used somewhat interchangeably.

The ordering primitive allows us to ask a natural question about brightness matching, namely about the symmetry of joint presentations:

$$
x \oplus u \sim u \oplus x,
$$

which is abbreviated as jp-symmetry (this is an analogue to the mathematical relationship $a+b=b+a$ ). In other words, is the image resulting from seeing a stimulus of intensity $x$ in the left eye and another of intensity $u$ in the right eye subjectively judged the same as that in the condition in which the stimuli are switched?

This test amounts to asking whether the two eyes are behaviorally identical and, formally, to asking whether the operator $\oplus$ is commutative. Whether this holds determines the next empirical steps; therefore, this is the first experiment. ${ }^{1}$

Generalized ratio production. A standard method in psychophysics (see Appendix B and, e.g., S. S. Stevens, 1975 , for a comprehensive discussion) is magnitude production. Popularized by S. S. Stevens (1975) and used for decades, magnitude estimation entails the respondent producing a stimulus that is some proportion of a standardfor example, twice as bright as a standard. The third primitive is a generalization of this magnitude production.

Suppose that $\mathrm{x}>y \geq 0$, and let $p>0$ be a real number. Let $(z, z)$ denote a signal pair that the respondent says makes the brightness interval ${ }^{2}$ from $(y, y)$ to $(z, z)$ stand in the ratio $p$ to the brightness interval from $(y, y)$ to $(x, x)$ (see Figure 3). Clearly, $z$ is a function of $x, y$, and $p$. It is convenient to write this function as a mathematical operator of the following form: $(x, x) \circ_{p}(y, y):=(z, z)$. This is called generalized ratio production or just ratio production. The generalization part of the ratio production can be seen by the fact that it agrees with ordinary magnitude production when $(y, y)=(0,0)$.

Notational convention. Let $\epsilon_{l}$ and $\epsilon_{r}$ denote thresholds for the left and the right eye, respectively, and let $x^{\prime}$ and $u^{\prime}$ be intensities of the stimuli presented in the left and the

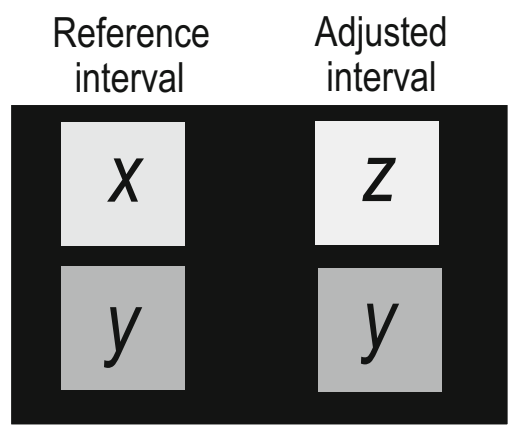

Figure 3. A stimulus in brightness ratio production. $x, y$, and $z$ are luminance levels. The respondents adjust the luminance of $z$ until they are satisfied that the brightness interval between $y$ and $z$ is a proportion $p$ of the interval between $y$ and $x$. The value of $p$ is indicated in the upper left corner of the monitor (not reproduced here).

right eye, respectively; then our notation is $x=x^{\prime}-\epsilon_{l}$ and $u=u^{\prime}-\epsilon_{r}$. Thus, $x=0$ denotes the threshold intensity (or less) of the left eye stimulus and $u=0$ denotes the same for the right eye. For signals well above threshold, which are used, the difference $x-x^{\prime}$ is negligible. Intensities are reported in $\mathrm{cd} / \mathrm{m}^{2}$.

\section{Representations of $\oplus$ and $\circ_{p}$}

Luce $(2002,2004)$ gave a set of necessary and sufficient (and testable) behavioral axioms, formulated in terms of the primitives, that allowed him to construct a numerical mapping $\Psi$, the psychophysical function, over the stimulus pairs that preserves the order $\succsim$ - that is,

$$
\Psi(x, u) \geq \Psi(y, v) \text { iff }(x, u) \succsim(y, v)
$$

- and for which there exists a constant $\delta \geq 0$ such that

$$
\Psi(x, u)=\Psi(x, 0)+\Psi(0, u)+\delta \Psi(x, 0) \Psi(0, u) .
$$

In addition, there is a strictly increasing numerical function $W$ from the positive real numbers onto the same domain such that

$$
\begin{aligned}
& W(p)=\frac{\Psi\left[(x, u) \circ_{p}(y, v)\right]-\Psi(y, v)}{\Psi(x, u)-\Psi(y, v)}, \\
& {[(x, u) \succ(y, v) \succsim(0,0)] .}
\end{aligned}
$$

The experiments focus on the form of Equation 6 where $u=x$ and $v=y$. There is no loss of generality from this choice (Luce, 2004).

In words, the order-preserving Condition 4 simply states that the brightness ordering judgment of the physical stimuli agrees with the physical intensity ordering. Property 5 captures the combination of inputs coming to the left and right eyes and is referred to as the summation representation (or sometimes as a $p$-additive representation in the literature). Property 6 describes the generalized ratio production operation and is referred to as the production representation.

There are two minor idealizations to note. First, these representations encompass the common idealization that $x, u$, and $y$ are any nonnegative real numbers. In practice, 
there is a maximum luminance level exposure that is safe for the human eye. Second, because of Fechner's paradox, the signal corresponding to, for example, $(x, 0)$ is really equivalent to some $\left(x^{\prime}, u^{\prime}\right)$ (evident from Figure 2), but since the research domain is restricted to the stimulus space outside of where Fechner's paradox obtains, this is not of concern.

The important point is that the representations map a subjective input onto a mathematical expression. That mathematical expression is indifferent to any biology and, in particular, nothing about any particular biology can be concluded from it. However, one strength is that it allows us to bring to bear the full force of the tools of mathematics to an unobservable subjective entity.

The representations consist of two unspecified functions, $\Psi$ and $W$, plus one constant. This allows for great freedom in capturing individual differences. Note that the functions and the representations are guaranteed to exist, provided that the parameter-free behavioral properties are satisfied. This situation is typical of axiomatic derivations of representations: no free parameters in the axioms and considerable freedom in the representations. Specifically, no fit of data to representations is performed, nor is any needed. For the reader who would like to delve into this issue, I recommend Appendix A as well as the comprehensive discussion of the general issue in Taagepera (2008).

Naturally, the actual mathematical forms of the unknown functions are of interest. In the axiomatic context, uncovering these forms entails formulating behavioral invariance properties that are equivalent to certain functional forms. Luce (2004) and Aczél and Luce (2007) formulated such properties, and Steingrimsson and Luce $(2006,2007)$ evaluated them for loudness. For the psychophysical function, $\Psi$, and the weighting function, $W$, they found support for power functions - or their generalization, called Prelec functions - for most respondents. Future work will be aimed at a parallel investigation for brightness. On the basis of decades of work on the form of the psychophysical function in a variety of domains (see S. S. Stevens, 1975, for an overview), that a power form might obtain would not be a surprise. Furthermore, given that $W$ is a cognitive function (relating, in all domains, a number to an intensity), one might speculate that it is not domain specific, and hence, should a power or a Prelec form obtain, it would not be a surprise either. The form of the functions is of no concern for the present investigation.

\section{Two Behavioral Properties of the Representations}

Now I turn to specifying the behavioral properties that will be tested and what can be concluded on the basis of their holding.

Subjective summation. From the results of Krantz, Luce, Suppes, and Tversky (1971), the necessary condition of binary additive conjoint measurement, the Thomsen condition,

$$
\left.\begin{array}{l}
x \oplus t \sim v \oplus w \\
v \oplus u \sim z \oplus t
\end{array}\right\} \Rightarrow x \oplus u \sim z \oplus w,
$$

must hold. In a qualitative sense, the Thomsen condition describes the additive cancellation of $t$ and $z$ (easily seen by substituting $\oplus$ with + and $\sim$ with $=$ ).

In the presence of some general background assumptions, ${ }^{3}$ the Thomsen condition (Equation 7) implies the summation representation (Equation 5). ${ }^{4}$ The Thomsen condition property is evaluated in Experiment 2.

Production commutativity. The basic idea embodied in the representation of generalized ratio production (Equation 6) is that the respondents perform the task as they are told to - that is, to produce a brightness difference that is some multiple of the difference in brightness of a standard stimulus. An important and easily demonstrated (Luce, 2002, 2004) consequence of Equation 6 is the behavioral property called (subjective) production commutativity: For $p>0$ and $q>0$,

$$
\left[(x, x) \circ_{p}(y, y)\right] \circ_{q}(y, y) \sim\left[(x, x) \circ_{q}(y, y)\right] \circ_{p}(y, y),
$$

where $y \geq 0$. Observe that the two sides differ only in the order of applying $p$ and $q$; that is, the test checks whether performing ratio production with $p$ and then with $q$ is equivalent to carrying out the same operation with the order of $p$ and $q$ switched. This is the reason for the word commutativity in its name. As was previously mentioned, production commutativity with $y=0$ also arose in $\mathrm{Na}$ rens's (1996) theory. That hypothesis was sustained for brightness by Peißner (1999) using $p, q>1$. In Experiment 3 , I evaluate the case of $y>0$.

\section{The Experimental Program}

The present experimental program is patterned on that carried out for loudness by Steingrimsson and Luce (2005a, 2005b, 2006, 2007). The present article tests the representations in Equations 5 and 6 separately. This means that, a priori, the $\Psi$ appearing in both representations is not guaranteed to be the same function. If one calls them $\Psi_{\oplus}$ and $\Psi_{o_{p}}$, a future second article will deal with the question of whether $\Psi_{\oplus}=\Psi_{\rho_{p}}=\Psi$. The results of the present article do not imply anything about this question. In a third article, the functional forms of the unknown functions $\Psi$ and $W$ will be explored.

\section{EXPERIMENTS 1-3}

Three experiments are presented: tests of jp-symmetry (Equation 3; Experiment 1), the Thomsen condition (Equation 7; Experiment 2), and production commutativity (Equation 8; Experiment 3).

\section{General Method}

The experiments reported here have a number of testing strategies in common that are now outlined. Other aspects are described later when relevant.

\section{Respondents}

A total of 21 students - graduate and undergraduate - from New York University and from the University of California, Irvine, and the author ${ }^{5}$ participated in the three experiments in this article; although it would have been desirable to do so, for practical reasons not all of the respondents participated in all of the experiments. All 
of the respondents reported normal or corrected-to-normal vision. All of the respondents, except the author, received compensation of $\$ 10$ per session.

\section{Stimuli}

The stimuli consisted of squares, subtending $10^{\circ}$ of visual angle, of achromatic light (RGB channels set to the same value) displayed on a computer monitor located in a dark room (see Figure 1A for an example).

\section{Apparatus}

The stimuli were generated with an Apple G4 computer using PsychToolbox extensions in MATLAB (Brainard, 1997; Pelli, 1997). At New York University, the stimuli were presented on a 17in. ViewSonic P810 CRT and at the University of California, Irvine, on an 18-in. NEC Multisync FE 950+, both with a resolution of $1,024 \times 768$ pixels and a refresh rate of $75 \mathrm{~Hz}$. The experiments were conducted in a dark, light-insulated room.

Equipment calibration and background conditions were of two kinds. A photometer, PhotoResearch PR-650, was used to measure luminance.

Calibration Condition 1 was done by averaging five repeated measures of luminance at every fifth value of the 255 RGB values, starting at 1 . The measures were taken from the left and right sides of the monitor, equidistant from its center. The luminance measures were fitted to a gamma function; the luminance disparity between the sides was not appreciable. For Condition 2, the procedure in Condition 1 was changed to better deal with possible spatial inhomogeneity in the monitor luminance output. ${ }^{6} \mathrm{~A}$ gamma function was determined for each monitor location where a stimulus was displayed. One of these was picked as a reference, and the RGB values for the other stimuli were determined to agree as closely as possible to a desired luminance using a reverse lookup procedure.

The monitors achieved an upper luminance of $\sim 100 \mathrm{~cd} / \mathrm{m}^{2}$, with the lowest stimulus level at $\sim 11 \mathrm{~cd} / \mathrm{m}^{2}$. Initially, the stimuli were displayed on a black (RGB values $0,0,0$ ) background. In order to minimize the mixing of scotopic and photopic conditions, the later experiments used $3.4 \mathrm{~cd} / \mathrm{m}^{2}$ background luminance, a level at which photopic vision is dominant (R. Blake, personal communication, September 12, 2007). This change largely coincides with the use of Calibration Condition 1. To maximize the available adjustment options, all available RGB values were used. ${ }^{7}$

In Experiments 1 and 2, a stereoscope (see Figure 1B) was used in the stimulus generation. A stereoscope uses a mirror system to project the left half of the monitor to the left eye (and vice versa). Placing a stimulus of intensity $x$ on the left side and one of intensity $u$ on the right side viewed through a stereoscope actualized the stimulus primitive $(x, u)$ (see the Joint Presentations section for details).

\section{Procedure}

The experiments were conducted in sessions lasting no more than $1 \mathrm{~h}$. The initial session was devoted to obtaining written consent, explaining the task, and running practice blocks. Depending on the experiment, practiced respondents typically completed around 60 estimates per session, organized into blocks of 6 or 8 estimates, presented in randomized order. Rest periods were encouraged, but their frequency and duration were under respondent control. The respondents received $10 \mathrm{~min}$ of dark adaption prior to each session. All of the respondents trained for one session on the task, except for those in Experiment 2, where training was for three sessions; it was determined in pilot studies that the respondents needed longer training before their data stopped showing large intersession variability. ${ }^{8}$

The summation operation, $\oplus$, and matches. The joint presentation $(x, u)$, which can also be written as $x \oplus u$, means that the stimulus $x$ is presented to the left eye and $u$ to the right eye. The goal is to obtain estimates of the subjective brightness match of joint presentation - that is, to find the stimulus $z \oplus z$ that is perceived as equal in brightness to $x \oplus u$; this can be written as $x \oplus u \sim z \oplus z$. Figures $1 \mathrm{~A}-1 \mathrm{C}$ describe the process. Figure $1 \mathrm{~A}$ depicts what was displayed on the monitor, where the letters indicate stimulus intensity. Figure 1B depicts the stereoscope through which the respondents view the monitor (see the Apparatus section). Figure 1C depicts what the participants saw. Since the stereoscope creates a cyclopic image, the percepts are those of $z \oplus z$ and $x \oplus u$.

To produce brightness matching, the respondents adjusted the intensity of $z$ until they were satisfied that the two percepts - the squares in Figure 1C-were equal in brightness. Specifically, the respondents used keypresses either to adjust the luminance of $z$ or to indicate their satisfaction with the brightness match. The respondents could choose any of four luminance steps (extra small, small, medium, large). After an adjustment, the screen was set to uniform background luminance for $100 \mathrm{msec}$, and then the next trial was presented. Subjectively, this was experienced as a blink and signaled that the adjustment had been made. This process was repeated until the respondents were satisfied with the match, indicated by a keypress, at which time the trial ended and $z$ was recorded as the response. Information about the current block and trial number was displayed in small letters in the upper left corner of the screen. In verbal instructions to the respondents, the task was explained as making the upper stimulus equal in brightness to the lower one.

Ratio productions, $o_{p}$. The goal of the ratio production is to obtain the estimate $z \oplus z=(x \oplus x) \circ_{p}(y \oplus y)$ (in shorthand, $\left.z=x \circ_{p} y\right)$. The task was to produce the intensity $z$ such that the difference in brightness between $y$ and $z$ was a proportion $p$ of the brightness difference between the reference signals $y$ and $x$.

Figure 3 depicts the stimulus as it was displayed on the monitor. The reference interval is on the left, from $y$ to $x$. The adjusted interval is on the right, where $y$ is reproduced and the respondent adjusts the intensity of $z$.

The adjustment procedure was the same as that for matching; the proportion $p$ was displayed on the upper left side of the monitor.

In the instructions to the respondents, the task was described as making the difference between the brightness of the lower and upper squares in the adjusted interval, for example, twice $(p=2)$ that between the reference squares on the left. The respondents were initially observed making the adjustments to help ensure their complete understanding of the task.

\section{Statistical Method and Presentation of Results}

Parameter-free null hypotheses of the form $L_{\text {side }}=R_{\text {side, }}$, which reflect the behavioral properties, are tested. As a matter of logic, a null hypothesis can never be proven empirically, but it can be empirically supported. As is common in physics, the goal of the statistical test is to establish a criterion by which the data can be said to support (or not) the null hypothesis.

If the hypothesis $L_{\text {side }}=R_{\text {side }}$ is correct, it is equivalent to asserting that both $L_{\text {side }}$ and $R_{\text {side }}$ are drawn from the same distribution. Yet, because there is no theory that predicts the distributions of the estimates, a nonparametric test (Mann-Whitney $U$ ) is used for statistical evaluation, with a significance level of $\alpha=.05$. This practice was used in similar studies (e.g., Ellermeier \& Faulhammer, 2000; Ellermeier, Narens, \& Dielmann, 2003; Steingrimsson \& Luce 2005a, 2005b, 2006, 2007; Zimmer, 2005; Zimmer, Luce, \& Ellermeier, 2001). Since intensity steps are discrete and estimates appear reasonably Gaussian, medians are best estimated by the mean, and variability by standard deviations. Hence, these are the central tendency indicators reported. Because we do not have an a priori model of how individuals relate, all data analysis is done on individual data (e.g., Luce, 1995).

To ensure that the sample sizes for $L_{\text {side }}$ and $R_{\text {side }}$ were sufficiently large to detect a true rejection of the null hypothesis, all statistical results were verified using Monte Carlo simulations based on the bootstrap technique. The question asked is whether $L_{\text {side }}$ and $R_{\text {side }}$ 
could, at the .05 level, be argued to come from the same underlying distribution. One thousand resamplings of the data were made, each subjected to the Mann-Whitney $U$ test, and the distribution of the results was examined (see Efron \& Tibshirani, 1993, and, in particular, Steingrimsson \& Luce, 2005a, for details). This was the criterion for acceptance of the null hypothesis, which would support the behavioral property.

Wilkinson and the Task Force on Statistical Inference (1999) included the recommendation of adding "brief comments that place ... effect sizes in a practical and theoretical context" (p. 599). Unfortunately, no practical guidelines were given for a nonparametric situation. Some effort has been made to tackle this problem, but I am not aware of any satisfactory solutions at the present time. One simple, yet powerful practical check on results lies in noting that should two medians (or means) differ by less than Weber's fraction, they are arguably not noticeably different to an observer. ${ }^{9}$ Teghtsoonian (1971) reported the mean Weber's fraction for brightness from five studies, all considered by him to be conservative, as .08 . As the reader can verify, in only one case (noted in context and the result considered marginal) do I fail to reject a null hypothesis in a case in which the means differ by more than this fraction. In fact, in several cases, the null hypothesis is rejected when the means differed by less than Weber's fraction, suggesting that the statistical criterion used for rejection was not lax.

\section{Experiment 1 Jp-Symmetry}

Because of results in audition (Steingrimsson \& Luce, 2005a), Luce (2004) expanded his (2002) theory to include the case where jp-symmetry fails. He termed this the biased case; the word bias simply means a deviation from jp-symmetry. Whether jp-symmetry holds determines which of somewhat different sets of behavioral properties must be tested. Thus, this is the natural first property to test.

\section{Method}

Testing the property involves obtaining two types of matches$x \oplus u=z \oplus z$ and $u \oplus x=z^{\prime} \oplus z^{\prime}$ - and statistically testing whether $z=z^{\prime}$. These matches were obtained as was described in the General Method section. Two luminance conditions were used; these are given in Table 1.

The three intensities in Table $1, a, b$, and $c$, gave rise to six ordered stimulus pairs - $(a, b),(a, c)$, and $(b, c)$, corresponding to the left side of Equation 3, and $(b, a),(c, a)$, and $(c, b)$, corresponding to the right side of Equation 3 - and three tests of jp-symmetry. These six matching conditions were all randomized within each block of trials.

\section{Results}

Fourteen individuals participated, and their data are presented graphically in Figure 4. Each graph shows the results of the six matching conditions, where, for example, the matching of $(x, u)$ is labeled $x u$ (multiplication is not implied, this only is a label) and the rest analogously.

Table 1

The Two Luminance Conditions, Luminance Levels (in $\mathbf{c d} / \mathbf{m}^{2}$ ), and Calibration Conditions in Experiment 1

\begin{tabular}{ccccc}
\hline \multirow{2}{*}{$\begin{array}{c}\text { Luminance } \\
\text { Condition }\end{array}$} & \multicolumn{3}{c}{ Luminance Level } & \\
\cline { 2 - 4 } & $a$ & $b$ & $c$ & Calibration \\
\hline $\mathrm{C}_{1}$ & 29.62 & 56.25 & 91.39 & Condition 1 \\
$\mathrm{C}_{2}$ & 15.05 & 35.22 & 66.07 & Condition 2 \\
\hline
\end{tabular}

The statistical hypothesis is that $(x, u) \sim(u, x) \Leftrightarrow x u=$ $u x$. Hence, using this labeling convention, the three statistical hypotheses to be tested are $a b=b a, a c=c a$, and $b c=c b$, which are marked on the abscissa. The average luminance level is marked on the ordinate. The sample size is indicated in the upper left portion of each graph.

Seven respondents (R1, R3, R5, R6, R8, R29, and R32) rejected all three testing conditions, 2 (R25 and R28) rejected two conditions, 4 (R7, R9, R27, and R30) rejected one, and 1 (R2) rejected none. Overall, jp-symmetry was rejected in 29 of the 42 tests.

For 13 of 14 respondents, one or more of the three conditions was found to be statistically different, with the trend for the remaining conditions consistent with this statistical difference. In 11 of the 14 respondents, the pattern of results was that of $(x, u) \succ(u, x)$, which in terms developed by Luce (2004) is called a right bias. For 2 respondents, weak evidence supports a left bias (R34 and R40). For the remaining respondent, the hypothesis of no bias - that is, $(x, u) \sim(u, x)$-was not rejected.

Luce's (2004) theory asserts nothing about the size or the direction of the deviation from symmetry (the bias). It does, however, assume monotonicity. Monotonicity would be violated if any of $a b<a c, b a<b c, c a<c b, b a<$ $b c$, or $c a<c b$ should fail (R. D. Luce, personal communication, April 2008). No violation of monotonicity was observed.

\section{Discussion}

The results echo those obtained for loudness: In general, jp-symmetry does not hold. But the results differ from those found previously in the sense that a right bias was predominant (there was weak support for two cases of left bias), whereas a majority of the respondents exhibited left bias in the auditory context (Steingrimsson \& Luce, 2005a).

Luce's $(2002,2004)$ theory admits bias in either direction, but the theory makes no attempt to explain the proportions of people who are left or right biased. Dominance of side (left vs. right) is a common human feature. Beyond the familiar handedness, most people exhibit eye dominance; but whether such dominance plays a part in the bias observed is an open question. Steingrimsson and Luce (2005a) explored one explanation-namely, that differences in thresholds of the two ears could explain the bias behavior. They rejected that explanation on the basis of the observation that in normal-hearing people, very small changes in energy were involved at the level of threshold relative to the energy in the wellabove-threshold stimuli used in their experiments. The disproportional energy differences are a consequence of loudness growing approximately as a power function of intensity. Since the situation is analogous for brightness, the same argument applies here. Understanding the reasons for the failure of jp-symmetry is of interest, but that is not the topic of this article, nor is it necessary for any of its conclusions. Thus, no further speculation is made with regard to this issue.

In conclusion, jp-symmetry (Equation 3) was not, as a general rule, found to hold for brightness. 


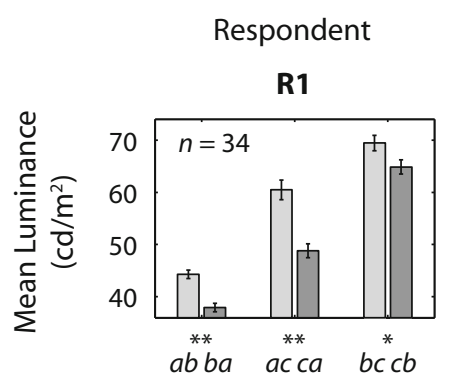

R6

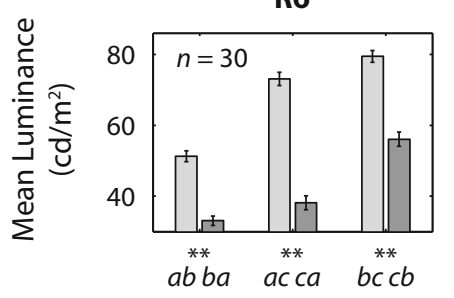

$\mathbf{R}^{\mathbf{5}}$

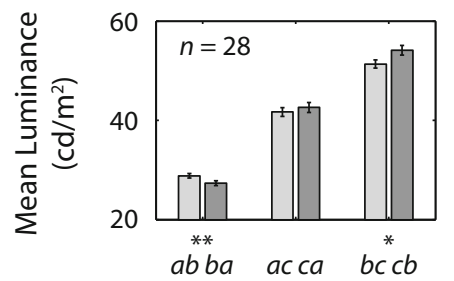

$\mathbf{R 3}^{\dagger}$

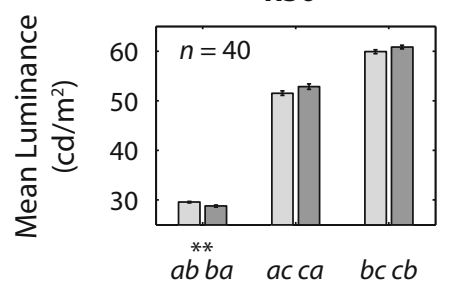

Condition
Respondent

R2

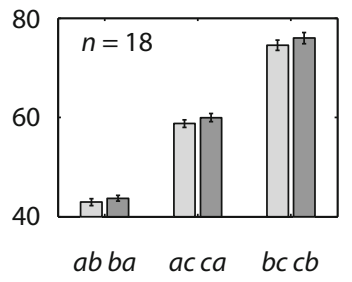

R7

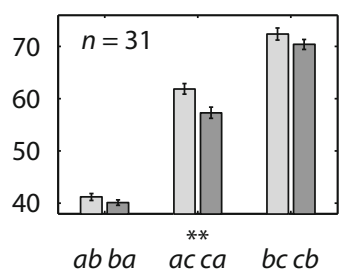

$\mathbf{R}^{\dagger}$

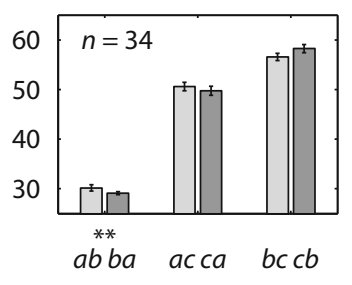

$\mathbf{R 3 2}^{\dagger}$

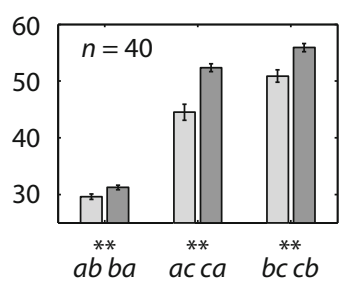

Condition
Respondent

R3

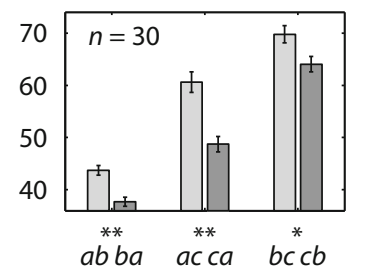

R8

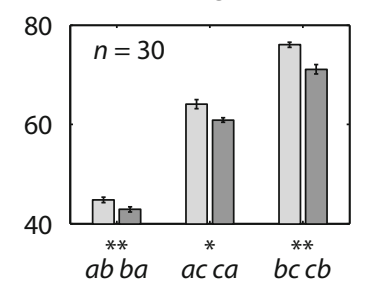

$\mathbf{R 2 8}^{\dagger}$

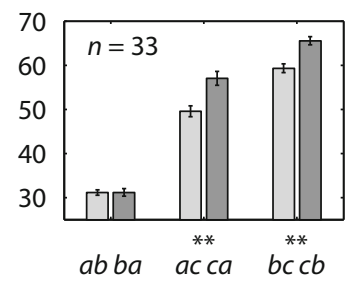

Condition
Respondent

R5

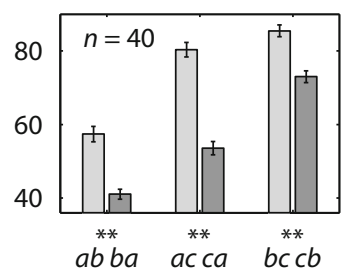

R9

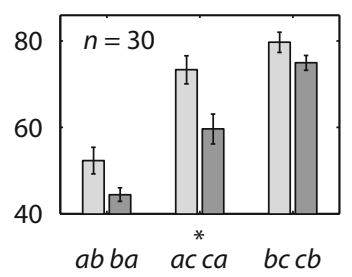

$\mathbf{R 2 9}^{\dagger}$

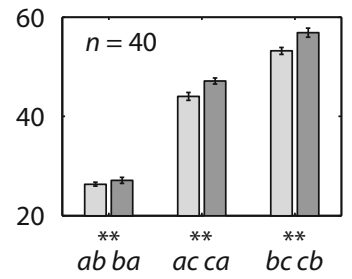

Condition

Figure 4. Experiment 1: Results from testing the jp-symmetry property, Equation 3. Error bars represent standard errors of the means. ${ }^{*} p \leq .05$. ${ }^{* *} p \leq .01$. (No * represents a failure to reject the null hypothesis.) ${ }^{*}$ Results from stimulus conditions $C_{2}$.

\section{Experiment 2 \\ Thomsen Condition}

The goal of Experiment 2 was to test the necessary condition of binary additive conjoint representation (Krantz et al., 1971, p. 250; Michell, 1990, pp. 68-73), the Thomsen condition.

To my knowledge, no test of the Thomsen condition (Equation 7); or of related properties has been carried out for brightness. Steingrimsson and Luce (2005a) studied several articles in which tests of the property were reported for audition. They then devised a testing method that involved binaural stimuli and three estimation steps in which the intensity was adjusted only in one ear. This feature initially produced larger than expected variability in respondents' judgments, a feature that receded with additional training. The results of pilot studies with brightness replicated this phenomenon, and in response, an additional four-step process was devised in which judgments were more balanced, in the sense that the trials featured conditions in which the luminance presented only to the left eye, only to the right eye, and to both eyes was adjusted. The results using both methods are reported.

\section{Method}

With reference to Equation 7 and the notation of Equation 1, the testing involved obtaining the estimates $z^{\prime}, w^{\prime}, y^{\prime}$, and $y^{\prime \prime}$ using the two methods in Table 2.

The testing requires that an estimate made in one trial be used as a stimulus in a subsequent trial. Steingrimsson and Luce (2005a) concluded that the best result was obtained when all estimates were collected within a session and the first estimate was used as the input in the subsequent estimation step. (See Appendices A.1, A.2, and A.4 in Steingrimsson \& Luce, 2005a, for details.)

The Thomsen condition is said to hold if $y^{\prime}$ and $y^{\prime \prime}$ are not found to be statistically different. 
Table 2

Trial Types for the Two Methods for Testing the Thomsen Condition (Experiment 2)

\begin{tabular}{cc}
\multicolumn{1}{c}{ Thomsen Condition (Experiment 2) } \\
\hline Method 1 & Method 2 \\
\hline$(x, t) \sim\left(v, w^{\prime}\right)$ & $(x, t) \sim\left(z^{\prime}, v\right)$ \\
$(v, u) \sim\left(z^{\prime}, t\right)$ & $\left(z^{\prime}, u\right) \sim\left(y^{\prime}, t\right)$ \\
$(x, u) \sim\left(y^{\prime}, y^{\prime}\right)$ & $(x, u) \sim\left(y^{\prime \prime}, v\right)$ \\
$\left(z^{\prime}, w^{\prime}\right) \sim\left(y^{\prime \prime}, y^{\prime \prime}\right)$ & \\
\hline
\end{tabular}

Table 3

Stimulus Conditions, Luminance Levels (in $\mathrm{cd} / \mathrm{m}^{2}$ ), Calibration Conditions, and Method Type (cf. Table 2) for Testing the Thomsen Condition (Experiment 2)

\begin{tabular}{|c|c|c|c|c|c|c|}
\hline \multirow{2}{*}{$\begin{array}{c}\text { Stimulus } \\
\text { Condition }\end{array}$} & \multicolumn{4}{|c|}{ Luminance Level } & \multirow[b]{2}{*}{ Calibration } & \multirow[b]{2}{*}{ Method } \\
\hline & $x$ & $t$ & $v$ & $u$ & & \\
\hline $\mathrm{C}_{1}$ & 44.58 & 25.31 & 11.48 & 69.29 & Condition 1 & 1 \\
\hline $\mathrm{C}_{2}$ & 43.11 & 24.43 & 10.88 & 66.91 & Condition 1 & 1 \\
\hline $\mathrm{C}_{3}$ & 34.21 & 29.91 & 17.28 & 58.36 & Condition 2 & 2 \\
\hline $\mathrm{C}_{4}$ & 26.09 & 23.09 & 13.28 & 40.49 & Condition 2 & 2 \\
\hline $\mathrm{C}_{5}$ & 25.95 & 35.22 & 40.49 & 30.37 & Condition 2 & 2 \\
\hline
\end{tabular}

All four/three trial types (see Table 2) were run twice within a block in a pseudorandomized order. Five stimulus conditions were used. These are given in Table 3 .

Two observations for each estimate were collected within a block of randomized trials.

\section{Results}

The results for 8 respondents are displayed in Table 4 . The Thomsen condition was not rejected for any of the 8 participants.

\section{Discussion}

The Thomsen condition is crucial to establish the summation representation (Equation 5). Whether brightness summates has been previously discussed, and the conclusions have varied (see Appendix C). Additivity (as is discussed in the Axiomatic literature; see Krantz et al., 1971, for details) implies that the brightness percept increases monotonically with luminance. Levelt (1965) tested this directly and showed just such monotonicity in the region in which Fechner's paradox does not obtain (see Figure 2). The present results suggest that this brightness increase is captured by the summation representation, Equation 5.

The reason for using two testing methods was the observation in pilot data that the respondents required substantially more training than in other tasks in order for intersession variability to stabilize; this is curiously parallel to the finding for loudness (Steingrimsson \& Luce, 2005a). Alas, I did not find that Method 1 solved the problem and eventually reverted to Method 2, which was quicker by virtue of having one fewer condition.

The Thomsen condition was found to hold for all 8 respondents, or in all 12 conditions. However, support was particularly weak for R25, with $p_{\text {stat }}$ values of .056 and .096 in Conditions $\mathrm{C}_{3}$ and $\mathrm{C}_{5}$, respectively, and in the latter case, the means differ by slightly more than Weber's fraction of .08. I will follow the criterion set above, but the support is really acceptable only for 7 of 8 respondents and in 10 of 12 conditions. However, that is quite good; hence, I conclude that the Thomsen condition has initial support in brightness.

\section{Experiment 3 Production Commutativity}

In principle, one would want to test the production commutativity property (Equation 8 ) for a variety of values of $p$ and $q$. In practice, the results of Steingrimsson and Luce (2007) suggest that the numerical distortion function, $W$ (Equation 6), differs for numbers above and below 1 and that, algebraically, nothing simple emerges from looking at the mixed case - for example, where $p<1, q>1$. Consequently, in practice, the cases of $p<1, q<1$ and $p>1$, $q>1$ are considered separately.

\section{Method}

The testing requires four estimates in two steps. The first step consists of estimating $v$ and then $w$ in

and

$$
(x, x) \circ_{p}(y, y) \sim(v, v)
$$

$$
(v, v) \circ_{q}(y, y) \sim(w, w),
$$

and the second of estimating $v^{\prime}$ and then $w^{\prime}$ in

and

$$
(x, x) \circ_{q}(y, y) \sim\left(v^{\prime}, v^{\prime}\right)
$$$$
\left(v, v^{\prime}\right) \circ_{p}(y, y) \sim\left(w^{\prime}, w^{\prime}\right) .
$$

\begin{tabular}{|c|c|c|c|c|c|c|c|c|}
\hline \multirow[b]{3}{*}{ Respondent } & \multirow{3}{*}{$\begin{array}{l}\text { Stimulus } \\
\text { Condition }\end{array}$} & \multicolumn{4}{|c|}{ Luminance Level } & \multirow[b]{3}{*}{$n$} & \multirow[b]{3}{*}{$p_{\text {stat }}$} & \multirow{3}{*}{$\begin{array}{c}\text { Statistical } \\
\text { Conclusion }\end{array}$} \\
\hline & & \multicolumn{2}{|c|}{$y^{\prime}$} & \multicolumn{2}{|c|}{$y^{\prime \prime}$} & & & \\
\hline & & $M$ & $S D$ & $M$ & $S D$ & & & \\
\hline R3 & $\mathrm{C}_{1}$ & 55.62 & 3.66 & 57.83 & 8.07 & 34 & .189 & $y^{\prime}=y^{\prime \prime}$ \\
\hline R4 & $\mathrm{C}_{1}$ & 56.71 & 2.30 & 57.40 & 4.31 & 30 & .917 & $y^{\prime}=y^{\prime \prime}$ \\
\hline R6 & $\mathrm{C}_{1}$ & 56.17 & 2.15 & 55.85 & 2.63 & 30 & .557 & $y^{\prime}=y^{\prime \prime}$ \\
\hline R9 & $\mathrm{C}_{1}$ & 51.89 & 2.76 & 52.13 & 5.45 & 30 & .976 & $y^{\prime}=y^{\prime \prime}$ \\
\hline $\mathrm{R} 14$ & $\mathrm{C}_{1}$ & 53.63 & 4.20 & 56.88 & 9.49 & 30 & .242 & $y^{\prime}=y^{\prime \prime}$ \\
\hline R8 & $\mathrm{C}_{2}$ & 52.91 & 2.75 & 53.97 & 4.53 & 30 & .445 & $y^{\prime}=y^{\prime \prime}$ \\
\hline $\mathrm{R} 8$ & $\mathrm{C}_{3}$ & 87.90 & 5.00 & 88.08 & 5.40 & 30 & .841 & $y^{\prime}=y^{\prime \prime}$ \\
\hline $\mathrm{R} 25$ & $\mathrm{C}_{3}$ & 84.74 & 9.07 & 79.07 & 13.09 & 30 & .056 & $y^{\prime}=y^{\prime \prime}$ \\
\hline R29 & $\mathrm{C}_{3}$ & 70.81 & 8.56 & 73.14 & 7.44 & 30 & .219 & $y^{\prime}=y^{\prime \prime}$ \\
\hline $\mathrm{R} 8$ & $\mathrm{C}_{4}$ & 65.96 & 5.26 & 63.78 & 4.46 & 30 & .136 & $y^{\prime}=y^{\prime \prime}$ \\
\hline R29 & $\mathrm{C}_{4}$ & 50.27 & 6.62 & 50.98 & 5.89 & 30 & .717 & $y^{\prime}=y^{\prime \prime}$ \\
\hline $\mathrm{R} 25$ & $\mathrm{C}_{5}$ & 49.77 & 14.29 & 44.35 & 8.10 & 30 & .096 & $y^{\prime}=y^{\prime \prime}$ \\
\hline
\end{tabular}

Table 4

Results From Testing the Thomsen Condition (Equation 7) in Experiment 2 
Table 5

Stimulus Types, Luminance Levels (in $\mathbf{c d} / \mathrm{m}^{2}$ ), and Proportions

\begin{tabular}{cccccccccc}
\hline & \multicolumn{2}{c}{$y$} & & \multicolumn{2}{c}{$x$} & & & \multicolumn{2}{c}{ Background } \\
\cline { 2 - 3 } \cline { 8 - 10 } Condition & NYU & UCI & & NYU & UCI & $p$ & $q$ & NYU & UCI \\
\hline $\mathrm{C}_{1}: p>1, q>1$ & 11.48 & 10.88 & & 29.62 & 28.62 & 2 & 3 & 0 & 3.4 \\
$\mathrm{C}_{2}: p<1, q<1$ & 11.48 & 10.88 & & 56.25 & 54.37 & $2 / 3$ & $1 / 3$ & 0 & 3.4 \\
\hline
\end{tabular}

Note-NYU, New York University; UCI, University of California Irvine.

Table 6

Results From Testing the Proportion Commutativity Property (Equation 8) in Experiment 3

\begin{tabular}{|c|c|c|c|c|c|c|c|c|}
\hline \multirow[b]{2}{*}{ Respondent } & \multirow[b]{2}{*}{ Condition } & \multicolumn{2}{|c|}{$w$} & \multicolumn{2}{|c|}{$w^{\prime}$} & \multirow[b]{2}{*}{$p_{\text {stat }}$} & \multirow[b]{2}{*}{$n$} & \multirow{2}{*}{$\begin{array}{l}\text { Statistical } \\
\text { Conclusion }\end{array}$} \\
\hline & & $M$ & $S D$ & $M$ & $S D$ & & & \\
\hline \multirow[t]{2}{*}{ R3 } & $\mathrm{C}_{1}$ & 67.05 & 18.74 & 69.16 & 15.00 & .643 & 32 & $w=w^{\prime}$ \\
\hline & $\mathrm{C}_{2}$ & 23.36 & 3.41 & 22.28 & 5.03 & .130 & 30 & $w=w^{\prime}$ \\
\hline \multirow[t]{2}{*}{$\mathrm{R} 4$} & $\mathrm{C}_{1}$ & 72.65 & 12.56 & 69.92 & 14.28 & .226 & 32 & $w=w^{\prime}$ \\
\hline & $\mathrm{C}_{2}$ & 17.82 & 1.57 & 19.38 & 1.78 & $<.001$ & 30 & $w \neq w^{\prime}$ \\
\hline \multirow[t]{2}{*}{$\mathrm{R} 8$} & $\mathrm{C}_{1}$ & 59.71 & 5.84 & 61.78 & 5.83 & .189 & 28 & $w=w^{\prime}$ \\
\hline & $\mathrm{C}_{2}$ & 17.74 & 3.62 & 17.03 & 3.07 & .490 & 28 & $w=w^{\prime}$ \\
\hline R11 & $\mathrm{C}_{2}$ & 17.39 & 6.27 & 16.80 & 3.73 & .979 & 32 & $w=w^{\prime}$ \\
\hline $\mathrm{R} 20$ & $\mathrm{C}_{2}$ & 19.80 & 1.35 & 20.27 & 1.82 & .176 & 40 & $w=w^{\prime}$ \\
\hline \multirow[t]{2}{*}{ R23 } & $\mathrm{C}_{1}$ & 68.95 & 6.49 & 68.99 & 7.24 & .733 & 30 & $w=w^{\prime}$ \\
\hline & $\mathrm{C}_{2}$ & 29.17 & 2.62 & 29.55 & 2.56 & .553 & 30 & $w=w^{\prime}$ \\
\hline \multirow[t]{2}{*}{$\mathrm{R} 4^{\dagger}$} & $\mathrm{C}_{1}$ & 71.39 & 11.78 & 69.28 & 13.56 & .189 & 32 & $w=w^{\prime}$ \\
\hline & $\mathrm{C}_{2}$ & 17.82 & 1.57 & 18.53 & 1.78 & .305 & 30 & $w=w^{\prime}$ \\
\hline \multirow[t]{2}{*}{$\mathrm{R} 8^{\dagger}$} & $\mathrm{C}_{1}$ & 62.66 & 6.44 & 59.73 & 5.37 & .221 & 32 & $w=w^{\prime}$ \\
\hline & $\mathrm{C}_{2}$ & 17.74 & 3.62 & 17.03 & 3.07 & .490 & 30 & $w=w^{\prime}$ \\
\hline \multirow[t]{2}{*}{$\mathrm{R} 31^{\dagger}$} & $\mathrm{C}_{2}$ & 70.40 & 13.11 & 71.65 & 9.65 & .801 & 30 & $w=w^{\prime}$ \\
\hline & $\mathrm{C}_{2}$ & 21.26 & 3.23 & 19.28 & 5.33 & .230 & 30 & $w=w^{\prime}$ \\
\hline \multirow[t]{2}{*}{$\mathrm{R} 33^{\dagger}$} & $\mathrm{C}_{1}$ & 72.30 & 9.03 & 69.33 & 9.91 & .121 & 30 & $w=w^{\prime}$ \\
\hline & $\mathrm{C}_{2}$ & 17.97 & 6.03 & 16.80 & 2.73 & .344 & 30 & $w=w^{\prime}$ \\
\hline
\end{tabular}

Data collected at NYU and using Calibration Condition 2; otherwise, data were collected at UCI and using Calibration Condition 1. Note: R4 and R8 ran in both locations.

Production commutativity is considered to hold if $w$ and $w^{\prime}$ are found not to differ statistically. Experiment 3 required a previous estimate to be used as input into a later estimate. Each individual estimate was used in subsequent estimates (see the Method section of Experiment 2 for more details). These matches are obtained as was described in the General Method section. Two observations for each estimate were collected within a block of randomized trials. Stimulus conditions are listed in Table 5.

\section{Results}

Ten respondents participated in this experiment. One participated only in Condition $\mathrm{C}_{1}$, and 2 respondents (data not presented) sought repeatedly to adjust their responses beyond the upper luminance limit of the monitor, making the respondents' desired adjustments inaccessible. The remaining data are presented in Table 6.

Table 6 lists respondent, condition, the means and standard deviations of $w$ and $w^{\prime}$, and results of the statistical tests. Conditions marked $\dagger$ mean that data were collected using Calibration Condition 2; the other data were collected with Calibration Condition 1. Note that for R4 and R8, the data were collected with both calibration methods. The only substantive difference is that although the property was rejected in $\mathrm{C}_{1}$ using the initial calibration, it was not rejected the second time around.

The production commutativity property was accepted in 17 of 18 tasks. ${ }^{10}$
Luce $(2002,2004)$ assumed monotonicity. Monotonicity here would be violated should judgments occur of $p=2>q=3$ and of $q=1 / 3>p=2 / 3$. That prediction was examined and was not violated in a single case (data not presented).

\section{Discussion}

Peißner (1999) investigated the related property, threshold-production commutativity - namely,

$$
\left[(x, x) \circ_{p}(0,0)\right] \circ_{q}(0,0) \sim\left[(x, x) \circ_{q}(0,0)\right] \circ_{p}(0,0),
$$

which is the special case of Equation 8 in which $y=0$. He used an experimental paradigm and stimuli similar to those employed here, except that he used only $p, q>1$ and found it to hold. The threshold-production commutativity property remains to be tested for $p, q<1$. Here the generalized proportion commutativity was tested. Given that it was sustained in 17 of 18 cases, it is concluded that the property has acceptable initial support in the brightness domain. This conclusion establishes the production representation (Equation 6).

\section{GENERAL DISCUSSION}

The topic has been a theory of global psychophysical judgments leading to the two representation classes. The 
Table 7

Summary of Experimental Results

\begin{tabular}{clccc}
\hline \multirow{2}{*}{ Experiment } & \multicolumn{1}{c}{ Po. of } \\
\cline { 2 - 5 } & \multicolumn{1}{c}{ Property } & $\begin{array}{c}\text { No. of } \\
\text { Tests }\end{array}$ & $\begin{array}{c}\text { No. of } \\
\text { Failures }\end{array}$ \\
\hline 2 & Jp-symmetry & 14 & 42 & 29 \\
3 & Promsen condition & 8 & 12 & 0 (2 close) \\
\hline
\end{tabular}

test results are summarized in Table 7. With a failure of jpsymmetry (Equation 3 ), the asymmetric case, the theory leads to the two representations in Equations 5 and 6, repeated here.

$$
\begin{aligned}
\Psi(x, u)= & \Psi(x, 0)+\Psi(0, u) \\
& +\delta \Psi(x, 0) \Psi(0, u),(\delta \geq 0),
\end{aligned}
$$

and

$$
\begin{aligned}
W(p)=\frac{\Psi\left[(x, u) \circ_{p}(y, v)\right]-\Psi(y, v)}{\Psi(x, u)-\Psi(y, v)}, \\
{[(x, u) \succ(y, v) \succsim(0,0)] . }
\end{aligned}
$$

The aim of this article was the separate testing of the properties derived from the first and second expressions. The results of the present article can, at best, support Equations 5 and 6 with different psychophysical functions, $\Psi_{\oplus}$ and $\Psi_{\circ_{p}}$ (which are possibly but not necessarily the same). Although ongoing work, not reported here, strongly supports the hypothesis that $\Psi_{\oplus}=\Psi_{\circ p}$, the overall conclusion from the three experiments reported here is that the summation and production forms of Luce's (2002) theory are separately supported in the brightness domain.

The present article parallels Steingrimsson and Luce (2005a) in form and result. This favorable outcome paves the way for a second article to parallel Steingrimsson and Luce (2005b). That article will evaluate the question of whether $\Psi_{\oplus}=\Psi_{\circ p}$.

Should that article provide favorable support for the theory, then Luce's $(2002,2004)$ theory seems to offer a certain unification on the level of description of at least these two domains. However, more work is needed before this statement can be made without reservation. Another worthwhile research avenue is to extend the work to other types of stimuli, monitors with larger luminance ranges, chromatic stimuli, and so on. Currently, all of these avenues are being explored.

\section{AUTHOR NOTE}

This research was supported in part by National Science Foundation Grants SBR-9808057 and BCS-0720288 (R. D. Luce, PI) to the University of California, Irvine; any opinions, findings, and conclusions or recommendations expressed in this material are those of the author and do not necessarily reflect the views of the National Science Foundation. Additional financial support was provided by the School of Social Sciences and the Department of Cognitive Sciences at UC Irvine. I am especially grateful to Marisa Carrasco for unfettered access to her laboratory and equipment at NYU to carry out much of the research reported here. I thank Randolph Blake and Jack Yellott for valuable input on technical matters. And I appreciate the many helpful comments of Joetta Gobell and R. Duncan Luce on earlier versions of the manuscript, as well as those of Michael Rudd who, in his role as a reviewer, made me work a lot but in return for a much improved article. Correspondence concerning this article should be addressed to R. Steingrimsson, Department of Cognitive Sciences, University of California, Irvine, CA 92697-5100 (e-mail: ragnar@uci.edu).

\section{REFERENCES}

AczéL, J., \& LucE, R. D. (2007). A behavioral condition for Prelec's weighting function on the positive line without assuming $\mathrm{W}(1)=1$. Journal of Mathematical Psychology, 51, 126-129.

BOLANOWSKI, S. J., JR. (1987). Contourless stimuli produce binocular brightness summation. Vision Research, 27, 1943-1951.

Bourassa, C. M., \& Rule, S. J. (1994). Binocular brightness: A suppression-summation trade off. Canadian Journal of Experimental Psychology, 48, 418-434.

Brainard, D. H. (1997). The Psychophysics Toolbox. Spatial Vision, 10, 433-436.

Cliff, N. (1992). Abstract measurement theory and the revolution that never happened. Psychological Science, 3, 186-190.

CoHn, T. E., \& LASLEY, D. J. (1976). Binocular vision: Two possible central interactions between signals from two eyes. Science, 192, 561-563.

CuRTis, D. W., \& RULE, S. J. (1978). Binocular processing of brightness information: A vector-sum model. Journal of Experimental Psychology: Human Perception \& Performance, 4, 132-143.

DE Silva, H. R., \& Bartley, S. H. (1930). Summation and subtraction of brightness in binocular perception. British Journal of Psychology, 20, 242-252.

DE Weert, C. M. M., \& Levelt, W. J. M. (1974). Binocular brightness combinations: Additive and nonadditive aspects. Perception \& Psychophysics, 15, 551-562.

Ding, J., \& Sperling, G. (2006). A gain-control theory of binocular combination. Proceedings of the National Academy of Sciences, $\mathbf{1 0 3}$, 1141-1146.

Efron, B., \& Tibshirani, R. J. (1993). An introduction to the bootstrap. New York: Chapman and Hall.

Ellermeier, W., \& FAulHammer, G. (2000). Empirical evaluation of axioms fundamental to Stevens's ratio-scaling approach: I. Loudness production. Perception \& Psychophysics, 62, 1505-1511.

Ellermeier, W., Narens, L., \& Dielmann, B. (2003). Perceptual ratios, differences, and the underlying scale. In B. Berglund \& E. Borg (Eds.), Fechner Day 2003: Proceedings of the 19th Annual Meeting of the International Society for Psychophysics (pp. 71-76). Stockholm: International Society for Psychophysics.

ENGEL, G. R. (1969). The autocorrelation function and binocular brightness mixing. Vision Research, 9, 1111-1130.

FALMAGNe, J.-C. (1976). Random conjoint measurement and loudness summation. Psychological Review, 83, 65-79.

Falmagne, J.-C., Iverson, G., \& Marcovici, S. (1979). Binaural "loudness" summation: Probabilistic theory and data. Psychological Review, 86, 25-43.

FECHNER, G. T. (1861). Über einige Verhältnisse des binocularen Sehens. Abhandlungen der mathematisch-physischen Classe der königlich sächsischen Gesellschaft der Wissenschaften, 5, 337-564.

Gigerenzer, G., \& Strube, G. (1983). Are there limits to binaural additivity of loudness? Journal of Experimental Psychology: Human Perception \& Performance, 9, 126-136.

Grossberg, S. (1997). Cortical dynamics of three-dimensional figureground perception of two-dimensional pictures. Psychological Review, 104, 618-658.

Grossberg, S., \& Kelly, F. (1999). Neural dynamics of binocular brightness perception. Vision Research, 39, 3796-3816.

IrTEL, H. (1986). Experimente zu Fechners Paradoxon der binokularen helligkeit. Zeitschrift für Experimentelle und Angewandte Psychologie, 33, 413-422. 
IRTEL, H. (1998). Binocular brightness combination: A mechanism for combining two sources of rather similar information. In W. G. K. Backhaus, R. Kliegl, \& J. S. Werner (Eds.), Color vision: Perspectives from different disciplines (pp. 267-274). Berlin: Walter de Gruyter.

Krantz, D. H., Luce, R. D., Suppes, P., \& TVersky, A. (1971). Foundations of measurement (Vol. 1). New York: Academic Press.

LEGGE, G. E. (1984). Binocular contrast summation-II. Quadratic summation. Vision Research, 24, 385-394.

LEHKY, S. R. (1983). A model of binocular brightness and binaural loudness perception in humans with general applications to nonlinear summation of sensory inputs. Biological Cybernetics, 49, 89-97.

LEVELT, W. J. M. (1965). Binocular brightness averaging and contour information. British Journal of Psychology, 56, 1-13.

Levelt, W. J. M., Riemersma, J. B., \& BunT, A. A. (1972). Binaural additivity of loudness. British Journal of Mathematical \& Statistical Psychology, 25, 51-68.

LUCE, R. D. (1995). Four tensions concerning mathematical modeling in psychology. Annual Reviews of Psychology, 46, 1-26.

LuCE, R. D. (2000). Utility of gains and losses: Measurement theoretical and experimental approaches. Mahwah, NJ: Erlbaum. (Errata: www .imbs.uci.edu/personnel/luce)

LUCE, R. D. (2002). A psychophysical theory of intensity proportions, joint presentations, and matches. Psychological Review, 109, 520-532.

LUCE, R. D. (2004). Symmetric and asymmetric matching of joint presentations. Psychological Review, 111, 446-454.

Luce, R. D., \& KrumhansL, C. L. (1988). Measurement, scaling, and psychophysics. In R. C. Atkinson, R. J. Herrnstein, G. Lindzey, \& R. D. Luce (Eds.), Stevens 'handbook of experimental psychology (2nd ed., Vol. 1, pp. 3-74). New York: Wiley.

LuCE, R. D., \& NARENS, L. (1993). Comments on the "non-revolution" in the representational theory of measurement. Psychological Science, 4, 127-130.

MacLeod, D. I. A. (1972). The Schrödinger equation in binocular brightness combination. Perception, 1, 321-324.

Michell, J. (1990). An introduction to the logic of psychological measurement. Hillsdale, NJ: Erlbaum

NARENs, L. (1985). Abstract measurement theory. Cambridge, MA: MIT Press.

NARENS, L. (1996). A theory of ratio magnitude estimation. Journal of Mathematical Psychology, 40, 109-129.

NARENS, L. (2002). A meaningful justification for the representational theory of measurement. Journal of Mathematical Psychology, 46, $746-768$.

NARENS, L. (2006). Symmetry, direct measurement, and Torgerson's conjecture. Journal of Mathematical Psychology, 50, 290-301.

PeißNer, M. (1999). Experimente zur direkten Skalierbarkeit von gesehenen Helligkeiten. Unpublished master's thesis, Universität Regensburg.

Pelli, D. G. (1997). The VideoToolbox software for visual psychophysics: Transforming numbers into movies. Spatial Vision, 10, 437-442.

Poletiek, F. (2001). Hypothesis-testing behaviour. Hove, U.K.: Psychology Press.

RoBerTs, F. S. (1979). Measurement theory with applications to decisionmaking, utility, and the social sciences. Reading, MA: AddisonWesley.

SCHNEIDER, B. (1988). The additivity of loudness across critical bands: A conjoint measurement approach. Perception \& Psychophysics, $\mathbf{4 3}$, 211-222

SChrodinger, E. (1926). Die Gesichtsempfindungen. In MuellerPouillets Lehrbuch der Physik (Book 2, Part 1, 11th ed., pp. 456-560). Vieweg: Braunschweig.

Steingrimsson, R., \& Luce, R. D. (2005a). Evaluating a model of global psychophysical judgments: I. Behavioral properties of summations and productions. Journal of Mathematical Psychology, 49, 290-307.

Steingrimsson, R., \& Luce, R. D. (2005b). Evaluating a model of global psychophysical judgments: II. Behavioral properties linking summations and productions. Journal of Mathematical Psychology, 49, 308-319.

Steingrimsson, R., \& Luce, R. D. (2006). Empirical evaluation of a model of global psychophysical judgments: III. A form for the psychophysical function and intensity filtering. Journal of Mathematical Psychology, 50, 15-29.

Steingrimsson, R., \& Luce, R. D. (2007). Empirical evaluation of a model of global psychophysical judgments: IV. Forms for the weighting function. Journal of Mathematical Psychology, 51, 29-44.

STEVENS, J. C. (1967). Brightness function: Binocular versus monocular stimulation. Perception \& Psychophysics, 2, 451-454.

Stevens, S. S. (1975). Psychophysics: Introduction to its perceptual, neural, and social prospects. New York: Wiley.

TAAGEPERA, R. (2008). Making social sciences more scientific: The need for predictive models. New York: Oxford University Press.

Teghtsoonian, R. (1971). On the exponents in Stevens' law and the constant in Ekman's law. Psychological Review, 78, 71-80.

Wade, N. J., \& ONO, H. (2005). From dichoptic to dichotic: Historical contrasts between binocular vision and binaural hearing. Perception \& Psychophysics, 34, 645-668.

Wilkinson, L., \& the Task Force on Statistical Inference (1999). Statistical methods in psychology journals: Guidelines and explanations. American Psychologist, 54, 594-604.

Zimmer, K. (2005). Examining the validity of numerical ratios in loudness fractionation. Perception \& Psychophysics, 67, 569-579.

Zimmer, K., Luce, R. D., \& Ellermeier, W. (2001). Testing a new theory of psychophysical scaling: Temporal loudness integration. In G. Sommerfeld, R. Kompass, \& T. Lachmann (Eds.), Fechner Day 2001: Proceedings of the 17th Annual Meeting of the International Society for Psychophysics. Lengerich, Germany: Pabst.

\section{NOTES}

1. The original impetus for Luce's (2002) model of global psychophysical judgments was a series of results originally developed within the context of utility theory (Luce, 2000). They were based on an assumption of commutativity of this operator, which in the psychophysical context could be translated into saying that, for example, the two eyes or ears are behaviorally identical. This hypothesis has been unambiguously rejected in audition (Steingrimsson \& Luce, 2005a) and, as we shall see, will be rejected for brightness as well (Experiment 1). The auditory data led Luce (2002) to generalize the result for the psychophysical context and to generalize further in Luce (2004)

2. The term interval is being used figuratively to refer to the difference in brightness that the respondents experienced between two intensity pairs.

3. Namely, monotonicity, solvability, and Archimedean-ness (a way of stating that subjectively measured intensities are commensurable).

4. The summation representation implies a stronger property called double cancellation. That property is the same as Equation 7, if each $\sim$ is replaced by $\succsim$. Obviously, double cancellation implies the Thomsen condition, but not the converse, except when solvability and monotonicity obtain (see Krantz et al., 1971, for details). This is of no consequence here.

5. This I judged acceptable because knowledge of the experimental design has no influence on the behavioral tasks of matching and ratio production. The author is numbered as $\mathrm{R} 8$.

6. Particular thanks go to M. Rudd who, as a reviewer, was responsible for this improvement in procedure.

7. Some researchers use linearized luminance steps. Since I sought subjective judgments from the respondents and because it is not a linear function of luminance, physical linearization does not clearly provide an advantage over finer adjustments. Subjective linearization is problematic because of individual differences.

8. The same phenomenon was observed in the analogous loudness experiment. The only hypothesis that I have for the behavior is that Experiment 3 is the only one in which intensity was adjusted in one ear/ eye rather than in both simultaneously, making the task-learning curve longer.

9. I thank J. Yellott for this simple and elegant observation.

10. R4's rejection for $\mathrm{C}_{2}$ seems surprising: $\mathrm{R} 4$ passed the same property in audition and also in pilot studies for brightness (with different equipment and evolving conditions). Yet, there was no objective reason to collect more experimental data, so the result stands. The respondent did, however, fail to reject this condition for a different set of stimuli. 


\section{APPENDIXA}

Theoretical Background

\section{In Defense of the Approach}

Taagepera (2008) discussed the prominent role that statistics plays in the social sciences. He observes that although the laws of physics were discovered without statistical hypothesis testing, such testing is a central tool in the social sciences. This observation led him to ask whether the laws of physics, such as the law of gravity $\left(F=G M m / r^{2}\right)$, might be uncovered by the statistical methods typically used by social scientists. To pursue this question, he produced a synthetic data set that fit the law of gravity (with a small random error) and sent it to 38 social scientists asking them if they could make sense of the relationship of the output variable $y$ to the input variables $x_{1}, x_{2}, x_{3}$ (he indicated having an idea of the relationship but refrained from telling them about it so as not to influence the outcome; see p. 19 for his precise wording). Although 8 individuals responded, none managed to uncover the law of gravity. However, all 8 did uncover high correlations of a variety of kinds, reporting $R^{2} \mathrm{~s}$ from .7 to .9 and even, in one case, as high as .98 (chap. 2). Taagepera noted that although these results are quite satisfactory by current social science norms (the positive and negative correlations were correctly identified, input factors were significant, and $R^{2}$ was high), all of the scientists failed to uncover the underlying pattern. This failure is likely due to their never having considered that a nonlinear relationship might underlie the data, in large part because the high $R^{2}$ did not seem to motivate a need for further analysis. Most laws in physics are nonlinear, yet in psychology, tests assuming linearity (e.g, ANOVA) are often used to the exclusion of exploring other possibilities. Although some reservations may be attached to Taagepera's "experiment," he raises the intriguing possibility that "[i]f some social phenomena did follow quantitative laws of the format most frequent in physics ... then the quantitative methods currently dominant in social sciences just might not suffice to discover them" (p. 20).

Comparing the kind of hypothesis testing typical in physics to that in psychology, it becomes clear that in physics, predictions of precise relationships are evaluated, entailing the nonrejection of a null hypothesis, whereas in contrast a typical experiment in psychology involves a hypothesis that some variable(s) is (are) not a factor and then proceeds to reject that null hypothesis (see Taagepera, 2008, and, e.g., Poletiek, 2001, chap. 1, for a comprehensive discussion).

There is an important difference between inferences that may be made on the basis of each of these two types of hypothesis testing. Support of a null hypothesis may assert exact relationships between variables and thereby provide for theories that are predictive and answer both how and how much a variable matters. In contrast, the information gleaned from rejection of a null hypothesis gives at best the information that a particular variable matters, but neither how nor by how much, nor does it result in a predictive theory.

Without going into what has been a long and complicated discussion in the philosophy of science, which largely harkens back to philosopher Karl Popper's theory of falsification, let it suffice to say that the (powerful) dominance of the theory of falsification in the social sciences to the exclusion of all other approaches, including the one that has given us modern physics, is, on the face of it, going a bit far. Thus, I suggest that the use of predictive models should at least be considered in the toolbox of the social sciences, and that the theory being evaluated here represents one use of this tool.

\section{The Axiomatic Measurement Approach}

Previous theoretical work in axiomatic psychophysics has used operations analogous to either the summation or production operations (e.g., Levelt, Riemersma, \& Bunt, 1972; Narens, 1996). However, the conceptual novelty in the psychological context (although typical in physics) is the linking of these two operations. This linking proved critical for establishing that a common psychophysical function can be used to represent both summation and production (currently being worked on in brightness).

The following is a much abbreviated account of the basic steps of axiomatic psychophysics (for a more extensive treatment, see, e.g., Krantz et al., 1971; Narens, 1985; Roberts, 1979). The axiomatizers tend to treat the following type of problem:

If a body of (potential) qualitative observations satisfies certain primitive laws - axioms that capture properties of these observations - then is it possible to find a numerical structure that accurately summarizes these observations? In technical terms, the question is: To which numerical structures is the set of qualitative observations isomorphic? An isomorphism is a one-to-one mapping between structures under which the structure of the one maps into that of the other. It is also desirable to have an explicit process whereby the numerical structure can be constructed from the qualitative one. (Luce \& Krumhansl, 1988, p. 5)

The axiomatic approach to this problem can be outlined as follows:

$[\mathrm{M}]$ easurement theory proceeds in the deductive fashion of mathematics: Certain formal properties are defined and theorems are proved. These theorems take the form of assertions that, if certain properties are true of the structure in question, then certain conclusions follow as a matter of pure logic. First, there are the primitive relations among attributes that determine both the measurement representations. ... Most results in measurement theory come in pairs. The first specifies conditions (axioms) under which it is possible to find numerical representation of the qualitative information. In other words, it formulates properties of a qualitative set of observations that are adequate for a certain kind of measurement system or scale to be appropriate. Such a result is called a representation theorem. The second type of result, called a uniqueness theorem, determines how unique the resulting measure or scale is. (Luce \& Krumhansl, 1988, p. 7) 


\section{APPENDIXA (Continued)}

Note that although this literature is purely mathematical, the choice of structures to be studied is much influenced by the intended application.

All measurement, whether within the axiomatic or the scaling tradition, begins with a method to study aspects of internal states such as brightness. One has, at present, few other options than to ask the respondents for an overt response - for example, which of two signals produces more or less of the psychological attribute in question (e.g., which is louder, brighter, etc.). (Recent development in fMRI and similar techniques may at some future date make it practical to observe neural activity in such a direct fashion that it becomes a meaningful direct measure of an internal response; for now, I must rely on overt responses.) For a variety of reasons, repeated questions of this kind tend to result in variability in responses, or what I take to be errors of measurement that must be dealt with statistically.

Note that the axiomatic approach has not escaped criticism. Perhaps the most serious of these criticisms is simply that, despite decades of work, it has produced little in terms of practical results (e.g., Cliff, 1992), although Luce and Narens (1993) countered this criticism, pointing to applications in utility theory and elsewhere. What seems clear is that in the field of psychophysics, practical applications of the axiomatic measurement approach have begun to emerge. The progress is both in theoretical articles that set forward directly testable behavioral axioms (e.g., Luce, 2002, 2004; Narens, 1996, 2002, 2006) and in empirical articles that report the results of such tests (e.g., Ellermeier \& Faulhammer, 2000; Ellermeier et al., 2003; Peißner, 1999; Steingrimsson \& Luce, 2005a, 2005b, 2006, 2007; Zimmer, 2005; Zimmer et al., 2001; the present article).

\section{Recent Directly Relevant Developments in Axiomatic Measurement}

Narens (1996) set out what he thought had to be the implicit assumptions behind S. S. Stevens's (1975) magnitude estimation/production methods. The first assumption was that respondents treated numbers in a veridical fashion. That is, if $W$ denotes a function that describes a respondent's interpretation of number $p$, then Narens (1996) showed that S. S. Stevens (1975) must have assumed $W(p)=p$. He formulated a behavioral axiom that was equivalent to a slightly weaker demand-namely, the power form $W(p)=p^{b}$. This property has been unambiguously rejected in audition (Ellermeier \& Faulhammer, 2000; Steingrimsson \& Luce, 2007; Zimmer, 2005) and in brightness (Peißner, 1999).

A second result of Narens (1996) was a behavioral axiom equivalent to asserting that measurements done using ratio productions are on a subscale of a ratio scale. This property has been tested for both loudness and brightness and has been generally sustained (Ellermeier \& Faulhammer, 2000; Peißner, 1999; Zimmer, 2005). Luce (2002) extended this result to what he called generalized ratio production and formulated the equivalent (subjective) production commutativity (Equation 8), which was supported in loudness by Steingrimsson and Luce (2005a). It is tested here in Experiment 3.

\section{APPENDIX B \\ Historical Context}

Psychophysicists typically study the relationship between subjective attributes and the physical stimuli from which they arise - in particular, how this changes as a function of changes in intensity. This is a measurement approach that is concerned not only with the assignment of numbers to sensations, but also with the formal properties of the number system into which the observed psychological measures are mapped. If the enterprise is successful, general laws that systematically relate sensations to physical attributes emerge. This is embodied, for example, in the famous laws of Fechner and Weber, as well as in the power law of S. S. Stevens (1975).

Central to S. S. Stevens's (1975) approach was his method of magnitude estimation/production, in which respondents were instructed to either give a number in response to a stimulus or to produce a stimulus that invoked a sensation that is in some prescribed proportion to a standard stimulus. The data collected using these methods suggest that for intensive continua (brightness, loudness, pain, heat, cold, etc.), sensation grows approximately as a power function of physical intensity (see S. S. Stevens, 1975, for a summary and references). These conclusions have almost exclusively involved averaging data over respondents, fitting the results to functional forms, and then evaluating the goodness of fit, where the power function form emerged as a well-fitting one.

Also studied in psychophysics is how intensity summates when, for example, signals are administered independently to the two ears (Falmagne, 1976; Falmagne, Iverson, \& Marcovici, 1979; Gigerenzer \& Strube, 1983; Levelt et al., 1972; Schneider, 1988; Steingrimsson \& Luce, 2005a, 2005b) or the two eyes (Bolanowski, 1987; Bourassa \& Rule, 1994; Cohn \& Lasley, 1976; Curtis \& Rule, 1978; de Silva \& Bartley, 1930; de Weert \& Levelt, 1974; Ding \& Sperling, 2006; Engel, 1969; Grossberg \& Kelly, 1999; Irtel, 1998; Levelt, 1965; J. C. Stevens, 1967) and how the two modalities compare (Lehky, 1983; Wade \& Ono, 2005). Summation has been studied in a variety of ways, all of which can be said to have in common that some comparison is made between sensation magnitudes when a signal intensity is varied in the two sensory organs. An example is sensation matching, which is the special case of ratio production in which the proportion is one. Respondents are typically instructed to produce a stimulus equal in subjective intensity to that of a standard. In the present experiments, the standard involves separately presenting lights of different intensities to the left and right eyes, and the respondent then matches the resulting sensation by adjusting the intensity of two other lights, also presented separately to the left and right eyes. 


\section{APPENDIX B (Continued)}

Few would deny the tremendous contribution that S. S. Stevens made to psychophysics. However, his approach has not been spared criticism. For the present purposes, it suffices to mention that S. S. Stevens (1975) never seems to have articulated or tested fundamental assumptions inherent in his magnitude estimation/production tasks. He appeared aware of some oddities that defied explanation; for example, he discussed what he termed a regression effect - namely, that magnitude estimations produced a fit to power functions that had slightly different exponents than did magnitude productions. He appeared to have assumed that respondents gave responses that preserved ratios and treated numbers in a veridical fashion. Lastly, he largely ignored individual differences, taking them to be noise in what he must have assumed was a universal mechanism.

Although this and similar approaches by those who may be called scalers have produced enormously useful information in a rather simple fashion, the problems just highlighted, as well as others not mentioned here, can be seen as motivation for the approach taken by the so-called axiomatizers, in whose form the theory being evaluated here is forged (Luce \& Krumhansl, 1988). These methods are detailed in Appendix A.

\section{APPENDIX C \\ Relation to Previous Work}

The task of comparing the present results to those in the existing literature would be a rather arduous one were it not for Grossberg and Kelly (1999). There is no compelling reason to do more than summarize their main conclusions; the reader interested in more detail on the literature is advised to start with Grossberg and Kelly.

Grossberg and Kelly (1999) evaluated and created a taxonomy of all the binocular summation models that they found to date (see their Table 1) and evaluated them against existing data. In addition, they presented an updated version of the FACADE theory (Grossberg, 1997).

The history of brightness summation has touched on topics ranging from whether it summates at all to how different stimuli produce different percepts. De Silva and Bartley (1930) wrote:

Numerous results gathered from observations of stereoscopic phenomena such as retinal rivalry support the view that alteration of conditions upon one retina always exerts some influence on the functioning of the other retina. Consequently it is rather surprising that Fechner, Sherrington, Abney and Watson, Dawson, and others have claimed to have proved by experiment that there is no binocular summation of brightness . . . as the weight of argument from the standpoint of numbers of investigations, prestige of investigators and use of refined apparatus is decidedly against it, binocular integration as regards brightness has come to be a generally discredited fact in the literature.

It was the purpose of this investigation to attempt to put this problem of binocular summation of brightness to a critical test, and in particular to determine if possible why the experimental results should be so flatly contradictory. (pp. 242-243)

I cannot help but find it somewhat ironic, some 77 years later, to be bringing up the question of brightness summation anew. Suffice it to say that the conclusion of de Silva and Bartley (1930), the aggregate conclusions of Grossberg and Kelly's (1999) review of additional literature, and the conclusion of the present article all support the idea that, outside the bounds of Fechner's paradox, brightness summates but that the effect is generally small.

Large summation effects have only been asserted for stimuli consisting of Ganzfelds (stimuli of uniform luminance that cover the entire visual field). Bolanowski (1987) reported, using magnitude estimation in flashed Ganzfeld conditions, that "complete binocular brightness summation occurs" (p. 1943). Bourassa and Rule (1994) reported two experiments, the first of which used Ganzfeld conditions and the second of which used smaller targets with very low spatial frequencies. They found that Ganzfeld stimuli "produced a large amount of binocular brightness summation and very little Fechner's paradox" (p. 418), whereas their smaller low-frequency stimuli "produced greater Fechner's paradox than the Ganzfelds, but more binocular summation and less Fechner's paradox than what is usually reported for small targets with abrupt contours" (p. 418).

Grossberg and Kelly (1999) identified 13 models, including the one that they proposed. Four of those they classified as eye weighting, 3 as vector summation, and 6 as neural networks. Grossberg and Kelly (1999, Section 5) meticulously dissected each of the proposed models and found a number of limitations that are applicable to the present discussion. They argued that the eye-weighting models of Levelt (1965) and Engel (1969) suffered from using weights that do not allow for binocular summation. They further argued that a model introduced by de Weert and Levelt (1974) made predictions that were at odds with the data. Their main problem with Irtel's (1986) model was that it did not seem to extend to Ganzfelds. This last argument seems too weak to reject the model outright. However, from the point of view of the present results, Irtel's (1986) model relies on an invariance condition that effectively assumes that the two eyes are identical, an assumption rejected here in Experiment 1. [For a reference in English, see Irtel (1998). However, note a typo where $\varepsilon(t x)$ and $m(t x)$ at threshold should equal $\varepsilon(x)$ and $m(x)$, respectively (H. Irtel, personal communication, August 10, 2006).] Whether that is a problem easily fixed is not something that is addressed here.

Grossberg and Kelly (1999) discussed several vector summation models (Curtis \& Rule, 1978; Legge, 1984; MacLeod, 1972; Schrodinger, 1926) and found flaws with them all, mostly related to their extendability to various stimulus conditions, such as Fechner's paradox. The latter criticism could also be leveled at the model tested here. Conversely, it seems that one would be going too far to reject these models on the grounds that they have 


\section{APPENDIX C (Continued)}

limitations, especially when it is untested whether those limitations may be overcome, and stimulus conditions under which Fechner's paradox obtains are not likely in natural contexts. In contrast, from the point of view of the present work, it seems that none of the models explicitly treat the biased case - that is, that the two eyes are different. It is not clear how hard it would be to amend these models to admit this empirical result, but for now, this appears to be a flaw.

The rest of the models identified and discussed by Grossberg and Kelly (1999) are neural network models. Although Grossberg and Kelly also found limitations in all of the neural network models that they evaluated, the main issue is, again, that these models treat the eyes as unbiased. However, it is not the intent here to reject every model proposed that reasonably accounts for data on the basis that they do not admit the biased case, especially since this situation may well be easily remedied.

Ding and Sperling's (2006) is a recent entrant into the group of neurally inspired models. Their approach is to divide various biological processes into black boxes and to model their input-to-output functions. An explicit goal of this form of modeling is to make it possible to reach into any black box and break it up into smaller boxes and thereby, incrementally, fashion a model that continues to become more detailed and accurate. This modeling effort shares the goal of the present one of enabling the accumulation of results. The model appears to assume that the eyes are identical, but it is likely an assumption that is easily adjusted. Of course, as the model is inspired by specific biology, it is not directly extendable to other domains, but the methodology may become an import avenue to incrementally evolve biologically inspired models.

However, as was emphasized in Appendix A, the goal is not to compete with or supplant existing models; the contrast drawn here is in the modeling approach. Luce $(2002,2004)$ took an approach that, in Grossberg and Kelly's (1999) and Ding and Sperling's (2006) framework, would define a new category - namely, an axiomatic model. Steingrimsson and Luce (2005a) articulated the main conceptual difference as follows:

First, [Luce's $(2002,2004)$ ] . . theory is not domain specific in the sense that it can, in principle, apply to any intensive dimensions (e.g., loudness, brightness, or [heaviness]). Second, although neuronal activity ultimately underlies perception, the approach taken here is entirely behavioral. The important abstraction is that the results we obtain are valid answers to our questions regardless of what the neural machinery may be, only the behavior matters. In effect, we could use the very same approach to an alien life form or a robot. Consequently, we do not make any attempt to draw conclusions about the biological workings of the perceptual system from our results. (p. 291)

For this reason, comparing neural network models to the model discussed here is not entirely apt. 